\title{
Prevalence, Pattern and Association of Pruritus with Quality of Life in Chronic Kidney Disease Patients Attending Kidney Care Centre, Ondo City, Southwest Nigeria
}

\author{
Oluseyi Adejumo', Ayodeji Akinbodewa ${ }^{1}$, Oladimeji Alli1, Abolarin Olatunji ${ }^{1}$, \\ Ifedayo Ibukun ${ }^{1}$
}

ABSTRACT

BACKGROUND: Pruritus is a common cutaneous manifestation of chronic kidney disease (CKD). It is associated with poor sleep quality, anxiety and depression which may contribute to reduction in quality of life (QoL). Paying more attention to pruritus in CKD patients may improve their QoL, reduce kidney disease burden and mortality. This study determined the prevalence, pattern and association of pruritus with QoL in CKD patients.

MATERIALS AND METHODS: This was a cross-sectional descriptive study carried out in a Kidney Care Centre, Ondo City, Southwest Nigeria. The severity and intensity of pruritus and health-related QoL were assessed using validated instruments. P-value of $<0.05$ was taken as significant.

RESULTS: There were 91 CKD subjects with a male:female ratio of 2.1:1. Sixty-nine (75.8\%) of the CKD patients were not on dialysis while the remaining twenty-two (24.2\%) were on maintenance hemodialysis (MHD). Thirty-nine (42.9\%) of the CKD subjects had pruritus which was mild in 25(64.1\%), moderate in $8(20.5 \%)$ and severe in 6(15.4\%). Pruritus was more common in MHD patients compared to predialysis $C K D$ patients $(50 \%$ vs $40.6 \%) p=0.47$. The median pruritus intensity score was also higher in MHD patients compared to predialysis CKD patients (40vs30) $p=0.51$. There was no significant association between gender, age, aetiology of CKD, stage of CKD and pruritus. There was a significant correlation between QoL score and pruritus intensity. $(p=<0.001, r=0.56)$.

CONCLUSION: Pruritus was common among our CKD subjects and it was not significantly associated with age, gender, stage or etiology of CKD. There was a significant association between impaired QoL and pruritus intensity.

KEYWORDS: Pruritus, quality of life, chronic kidney disease

DOI: http://dx.doi.org/10.4314/ejhs.v26i6.7

\section{INTRODUCTION}

Pruritus is one of the common cutaneous manifestation of chronic kidney disease (CKD) that is present in $40-84 \%$ of end stage renal disease population (1-5). There is variation in its distribution and severity. Uremic pruritus frequently affects localized parts of the body such as face, chest, back, limbs and may be generalized in up to $50 \%$ of those affected (6). It may affect both predialysis CKD patients and those already on renal replacement therapy. There is no significant difference between the prevalence of pruritus in those on hemodialysis (HD) or peritoneal dialysis (PD) (1).

The exact pathogenesis of pruritus in CKD is unknown. However, some pathogenetic mechanisms have been suggested. These include divalent ion abnormalities such as calcium and magnesium, phosphate abnormalities, hyperparathyroidism, iron deficiency anaemia, xerosis, increased cutaneous mast cells and uremic toxins $(7-10)$.

${ }^{1}$ Kidney Care Centre, University of Medical Science, Ondo State, Nigeria

Corresponding Author: Oluseyi A. Adejumo, Email: ceeward2010@yahoo.com 
Pruritus in CKD patients is associated with poor sleep, anxiety and depression which may contribute to reduction in overall quality of life (QoL) in these patients $(1-3,11,12)$. Pruritus is also associated with increased mortality in CKD patients $(3,12)$. There are therapeutic interventions useful in treatment or reduction of the severity of uremic pruritus such as improvement in adequacy of dialysis, treatment of calcium, phosphate abnormalities, correction of iron deficiency and use of ultraviolet B light (10,13-15). Therefore, paying more attention to pruritus in these patients may improve their QoL, reduce kidney disease burden and mortality. There is still a major gap in the knowledge of pruritus and its association with quality of life in CKD patients in Nigeria, hence the need for this study which aimed:

1. To determine the prevalence and pattern of pruritus in CKD patients attending Kidney Care Centre, Ondo City, Southwest Nigeria.

2. To determine the association between pruritus severity and QoL in these CKD patients.

\section{MATEIALS AND METHODS}

Study design: This was a descriptive crosssectional study carried out in the Kidney Care Centre, Ondo City which is a state-government owned tertiary hospital located in Southwest Nigeria. The study was conducted between September 2015 and May 2016.

Sample size and study population: The minimum sample size for this study was 90 after including 10\% attrition rate using Epi Info sample size calculator for a population that is less than 10,000. The prevalence of pruritus in CKD patients was taken as $26.2 \%$ from a previous study and $95 \%$ was used as the confidence interval (16). However, a total of $91 \mathrm{CKD}$ subjects were recruited for the study. Adult predialysis CKD and maintenance HD patients were included in the study. Those with with primary skin disorder and immunosuppressant therapy were excluded.

The effect of pruritus on QoL of the CKD subjects was assessed using 'Skindex-10' questionnaire which had been previously validated for uremic pruritus (5). This questionnaire consists of ten questions and each has five options as answer (never, rarely, sometimes, often and all the time which were scored as 0,1,2,3 and 4 respectively in this study). This questionnaire assessed sleep, social function and emotion domains. The summation of these scores gave the QoL score for each patient with pruritus. A "SelfAssessed Disease Severity" questionnaire which allowed patients to categorize themselves into mild, moderate and severe, depending on severity of concomitant signs and symptoms was used to assess pruritus severity. This self-categorization instrument had been previously validated and found useful to predict intensity and chronicity of uremic pruritus (17). For itching intensity, a 100$\mathrm{mm}$ visual analog scale (VAS) was used to assess pruritus intensity.

All study subjects were interviewed using an interviewer administered questionnaire after taking informed consent. This questionnaire had sections for demographic data, etiology of CKD, pattern of pruritus, severity of pruritus and effect of pruritus on QoL of the study subjects. The patients were staged according to Kidney Disease Improving Global Outcome guidelines (18).

Data analysis: Data was analyzed using the statistical package for social sciences (SPSS) version 17.0. Results were presented in tabular form. Univariate analysis was used in description of the characteristics of the study population. Discrete variables were presented as frequency and percentages. Continuous variables were presented as median, interquartile range while Mann Whitney U was used to compare the median within sub-groups. Chi-square test was used to determine the significance of observed differences for categorical variables. Spearman's correlation was used to determine association between QoL score and pruritus intensity. $\mathrm{P}$ value $<0.05$ was considered significant.

\section{RESULTS}

The study population consisted of 91 CKD subjects with 62 males $(68.1 \%)$ and 29 females $(31.9 \%)$. The majority of the subjects were less than 65 years, accounting for $76(83.6 \%)$ of the total population. Twenty-two $(24.2 \%)$ of the CKD were on maintenance hemodialysis (MHD), $53(58.2 \%)$ in CKD stage 5, 10(11.0\%) in CKD stage 4 and $6(6.6 \%)$ in CKD stage 3 . The common etiologies of CKD in the study were chronic 
glomerulonephritis in $33(36.2 \%)$, hypertension in $24(26.4 \%)$ and diabetes mellitus in $17(18.7 \%)$. Thirty-nine $(42.9 \%)$ of the CKD subjects had pruritus. The intensity of pruritus according to self categorization was mild in $25(64.1 \%)$, moderate in $8(20.5 \%)$ and severe in the remaining $6(15.4 \%)$ (Table 1).

Table 1: Characteristic of Study population.

\begin{tabular}{lc}
\hline \multicolumn{1}{c}{ Parameters } & $\mathbf{n}(\%)$ \\
\hline Age & \\
$<45$ years & $39(42.9)$ \\
$45-64$ years & $37(40.7)$ \\
$\geq 65$ years & $15(16.4)$ \\
Gender & \\
Male & $62(68.1)$ \\
Female & $29(31.9)$ \\
CKD stage & \\
3 & $6(6.6)$ \\
4 & $10(11.0)$ \\
5 & $53(58.2)$ \\
$5 \dagger \quad 52(24.2)$ \\
Aetiology of CKD & \\
CGN & $33(36.2)$ \\
DM & $17(18.7)$ \\
Hypertension & $24(26.4)$ \\
Others & $17(18.7)$ \\
Pruritus & \\
Present & $39(42.9)$ \\
Absent & $52(57.1)$ \\
Pruritus Intensity & \\
Mild & $25(64.1)$ \\
Moderate & $8(20.5)$ \\
Severe & $6(15.4)$ \\
\hline CKD (chronic kidney & disease) CGN (chronic \\
glomerulonephritis), DM & (diabetes mellitus), 5† \\
(Maintenance HD) & \\
\hline
\end{tabular}

Pruritus was present in 25(40.3\%) of the male CKD subjects which was lower compared to $14(48.3 \%)$ of the female CKD subjects, although this was not significant $(\mathrm{p}=0.51)$. Pruritus was more common in those with CKD from chronic glomerulonephritis and diabetes mellitus compared to other etiologies. Pruritus was present in $18(45 \%)$ of young CKD subjects, $17(44.7 \%)$ of middle aged CKD subjects and 6(33.3\%) of the elderly CKD subjects. Although pruritus was more common in young and middle aged CKD subjects compared to elderly subjects. This was however not significant ( $\mathrm{p}=0.38$ ) (Table 2).

The proportion of CKD patients on MHD with pruritus was higher compared to predialysis CKD patients (50\% vs $40.6 \%)$, although not statistically significant $(\mathrm{p}=0.47)$. There was no significant association between gender, age, aetiology of $\mathrm{CKD}$, stage of $\mathrm{CKD}$ and pruritus (Table 2).

The median VAS of pruritus intensity of predialysis CKD patients was 30(28). This was lower compared to 40(35) in MHD patients. However, this was not statistically significant $(\mathrm{p}=0.51)$.

Pruritus was generalized in $11(28.2 \%)$ of the CKD subjects. The commonly affected localized areas were the limbs in $10(25.7 \%)$, trunk in $9(23.1 \%)$ and a combination of limbs and trunk in 7(18\%) (Fig 1). There was a significant positive correlation between QoL score and visual analogue score for pruritus intensity $(\mathrm{p}=<0.001$, $\mathrm{r}=0.56$ ) (Fig 2). 


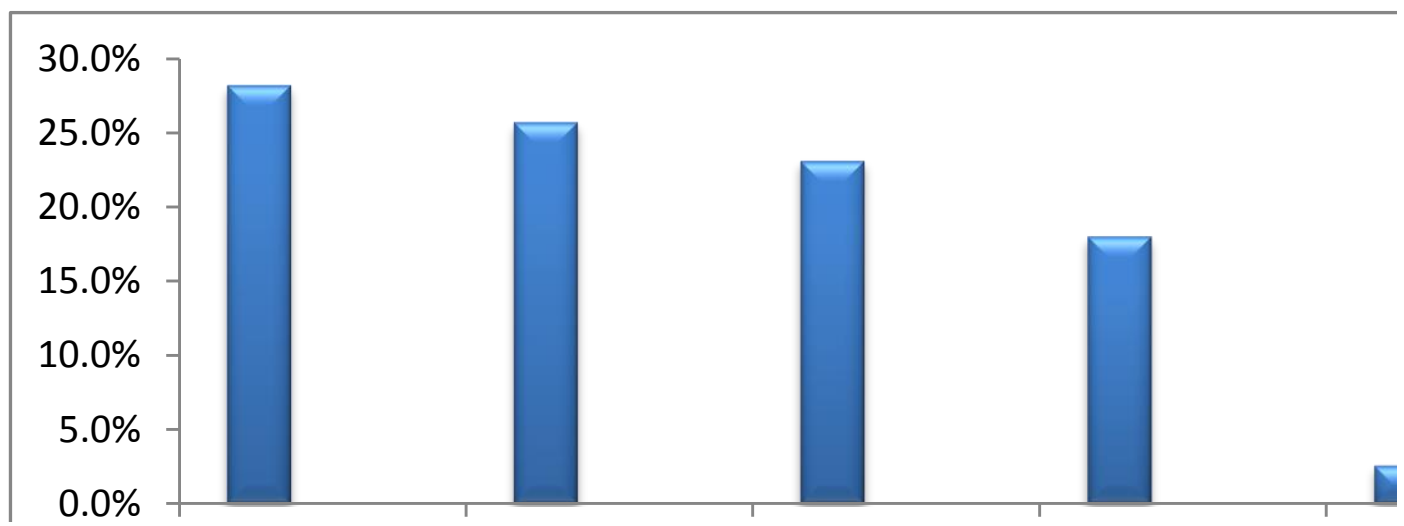

Figure 1: Frequency of body parts affected by Pruritus

Table 2: Association between Pruritus, age, aetiology and stage of CKD

\begin{tabular}{llcc}
\hline & Pruritus Present n(\%) & Pruritus Absent n(\%) & P-value \\
\hline Gender & & & \\
Male & $25(40.3)$ & $37(59.7)$ & 0.51 \\
Female & $14(48.3)$ & $15(51.7)$ & \\
Age & & & \\
<45 years & $18(45.0)$ & $22(55.0)$ & 0.38 \\
45-64 years & $17(44.7)$ & $21(55.3)$ & \\
$\geq 65$ years & $4(30.8)$ & $9(69.2)$ & \\
CKD Aetiology & & & \\
CGN & $15(45.5)$ & $18(54.5 \%)$ & \\
Hypertension & $7(29.2)$ & $17(70.8)$ & \\
DM & $11(64.7)$ & $6(35.3)$ & \\
Others & $6(33.3 \%)$ & $11(66.7 \%)$ & \\
CKD STAGE & $28(40.6)$ & $41(59.4)$ & $11(50.0)$ \\
Predialysis & $11(50.0)$ & DM(diabetes & mellitus) \\
MHD & $C G N($ chronic glomerulonephritis)
\end{tabular}
(Maintenance Hemodialysis)

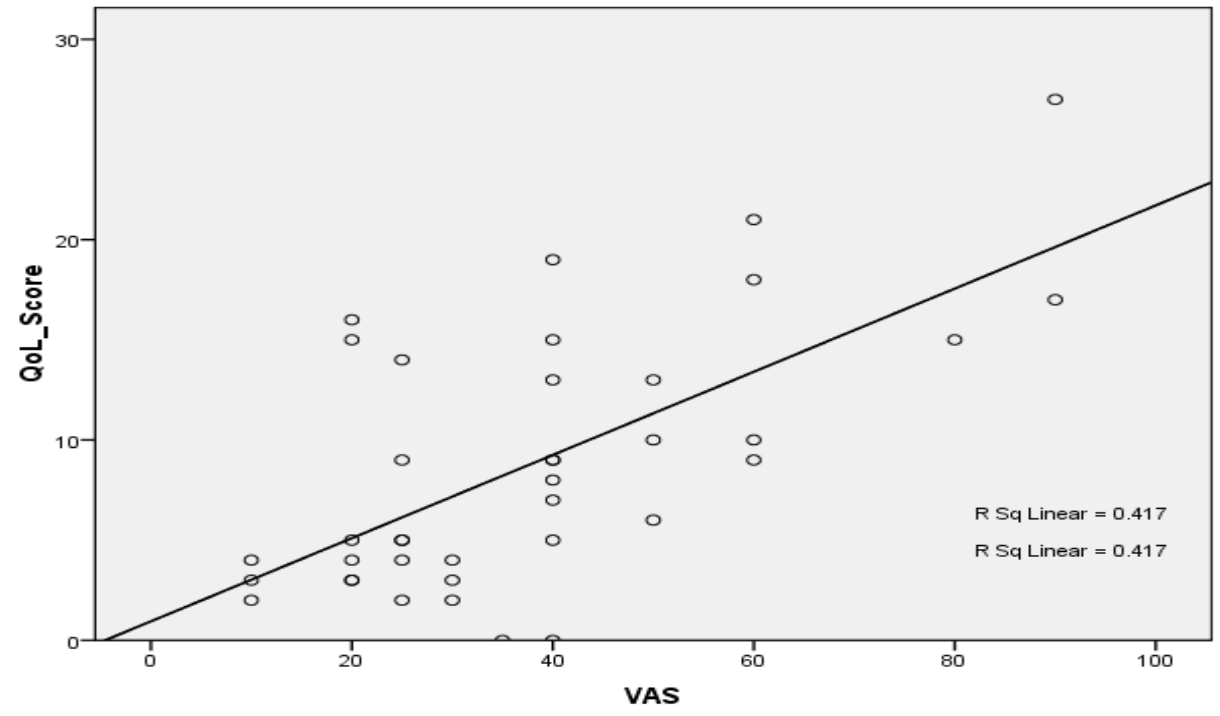

Figure 2: Correlation between Pruritus intensity and quality of life score 


\section{DISCUSSION}

This study showed that a significant proportion of both MHD and predialysis patients had pruritus There was also significant association between QoL score and pruritus intensity among our CKD subjects.

The prevalence of pruritus in this study is $42.9 \%$ which is higher than $18.9 \%$ reported by Solak et al (19). The higher prevalence in our study may be due to the fact that the study subjects consisted of both predialysis CKD and MHD patients unlike those in the study by Solak et al who were only predialysis patients. Tessari et al and Marthur et al however reported a higher prevalence of $52.1 \%$ and $84 \%$ respectively compared to our study. This may be due to the fact that all their study subjects were on MHD unlike this study $(1,5)$.

There was no significant difference in th e occurrence of pruritus in relation to age, gender, CKD stage and aetiology of CKD. This is similar to findings reported by Solak et al and Berger et al $(19,20)$. This implies that pruritus should be assessed and managed in all CKD patients irrespective of age, gender, stage or etiology.

In this study, the prevalence of pruritus was higher in MHD patients compared to the predialysis patients. The intensity of pruritus was also more severe in MHD patients. These may be related to higher level of inflammatory markers usually observed in HD subjects which have also been implicated in the pathogenesis of uremic pruritus (21-23).

This study also showed that pruritus affected the lower limbs and trunk most commonly which is similar to findings of other previous studies $(6,24)$. The pattern of pruritus severity determined by self-assessment showed that the majority of the CKD subjects in this study had mild to moderate pruritus which is different from findings by Marthur et al who reported that there were more patients with moderate to severe pruritus in their study (5).This may also be explained by the fact that all patients in the latter study were on HD and were more likely to have severe form of pruritus.

There was a significant association between intensity of pruritus assessed by VAS and quality of life scores which is similar to findings in other studies $(1,3-5,25)$. This shows that the quality of life of our CKD patients is adversely affected with increasing intensity of pruritus. Pruritus has also been reported to be associated with higher mortality risk in CKD patients $(3,13)$. Therefore, clinicians should pay more attention to pruritus in CKD because of its overall negative impact on quality of life and mortality. VAS is a simple and quick method of assessing severity of pruritus which may be used to follow up the effect of therapeutic interventions instituted to manage pruritus in CKD patients.

Correction of anemia, calcium-phosphate abnormalities and optimizing dialysis may reduce severity of pruritus $(10,13,15)$. This may therefore improve QoL and reduce mortality of CKD patients. The limitation of this study is that it is a single centre study whose findings cannot be generalized. Also, calcum-phosphate abnormalities which may have association with pruritus were not assessed in this study.

In conclusion, pruritus was common among our CKD subjects, and it was not associated with age, gender, stage or aetiology of CKD. There was a significant association between QoL score and pruritus intensity. Therefore, treating pruritus may improve QoL of CKD patients.

Pruritus should be regularly assessed and effectively managed in CKD patients in order to reduce associated morbidity, mortality and improve overall QoL.

\section{REFERENCES}

1. Tessari G, DalleVedove C, Loschiavo C, Tessitore N, Rugiu C, Lupo A et al.The impact of pruritus on the quality of life of patients undergoing dialysis: a single centre cohort study. J Nephrol. 2009;22(2):241-248.

2. Zucker I, Yosipovitch G, David M, Gafter U, Boner G..Prevalence and characterization of uremic pruritus in patients undergoing hemodialysis: uremic pruritus is still a major problem for patients with end-stage renal disease. $J$ Am Acad Dermatol. 2003 ;49(5):842-846.

3. Pisoni RL, Wikström B, Elder SJ, Akizawa T, Asano Y, Keen ML et al Pruritus in haemodialysis patients: International results from the Dialysis Outcomes and Practice Patterns Study (DOPPS). Nephrol. Dial Transplant 2006:21(12):3495-3505. 
4. Susel J, Batycka-Baran A, Reich A, Szeipetowski JC. Uremic pruritus markedly affects quality of life and depressive symptoms in hemodialysis patients with end stage renal dsease. Acta Derm Venerol 2014;94(3):276-278.

5. Mathur VS, Lindberg J, GermainM,Block G, Tumlin J, Smith M et al. A longitudinal study of uremic pruritus in hemodialysis patients. Clin J Am SocNephrol 2010;5(8):1410-1419

6. Mettang T, Pauli-Magnus C, Alscher DM. Uremic pruritus-new perspectivesand insights from recent trials. Nephrol Dial Transplant 2002;17:1558-1563.

7. Hiroshige $\mathrm{K}$, Kabashima $\mathrm{N}$, Takasugi $\mathrm{M}$, Kuroiwa A. Optimal dialysis improves uremic pruritus. Am J Kidney Dis 1995;25:413-419.

8. Virga G, Visentin I, La Milia V, Bonadonna A. Inflammation and pruritus in haemodialysis patients. Nephrol Dial Transplant 2002;17:2164-2169.

9. Blachley JD, Blankenship M, Menter A, Parker T III, Knochel JP. Uremic pruritus: skin divalent ion content and response to ultraviolet phototherapy. Am J Kidney Dis 1985;5:237-241.

10. Hiroshige K, Kabashima N, Takasugi M, Kuroiwa A. Optimal dialysis improves uremic pruritus. Am J Kidney Dis 1995;25:413-41

11. Lopes GB, Nogueira FC, de Souza MR, Penalva MA, de Amorim JL, Pisoni RL et al. Assessment of the psychological burden associated with pruritus in hemodialysis patients using the kidney disease quality of life short form. Qual Life Res 2012;21(4):603612.

12. KimataN, Fuller DS, Saitu A, Akizawa T, Fukuhara S, Pisoni RL et al Pruritus in hemodialysis patients:Results from Japanese Dialysis Outcomes and Practice Patterns Study (JDOPPS). Hemodial Int 2014;18(3):657-667.

13. Chen ZJ,CaoG,Tang WX, Lv XY, Huang SM, Qin $\mathrm{W}$ et al. A randomized controlled-trial of high permeability haemodialysis against conventional haemodialysis in treatment of uremic pruritus. Clin Exp Dermatol 2009;34:679-683.

14. Ada S, Seçkin D, Budakoğlu I, Ozdemir FN. Treatment ofuremic pruritus with narrow band ultraviolet B phototherapy: an open pilot study. J Am AcadDermatol. 2005;53(1):14951.

15. Noordzij M, BoeschotenEW,Bos WJ, Dekker FW, Bossuyt PM, Krediet RT et al. Disturbed mineral metabolism is associated with muscle and skin complaints in a prospective cohort of dialysis patients. Nephrol Dial Transpl 2007;22;2944-2949.

16. Falodun O, Ogunbyi A, Salako B, George AK. Skin changes in patientswth chronic renal failure. Saudi $J$ Kidney Dis Transpl 2011;22:268-272.

17. Bhaduri S, Mathur V, Fellmann J, Rosen D. Uremic puritus patients: A national survey of sleep and mood disruption. Am J Kid Dis 2007; 49:31.

18. National Kidney Foundation. KDIGO 2012 Clinical Practice Guideline for the Evaluation and Management of Chronic Kidney Disease. Kidney Int Suppl 2013;3:19-62.

19. Solak B, Acikgoz SB, Sipahi S, Erdem T. Epidemiology and determinants of pruritus in pre-dialysis chronic kidney disease patients. Int Urol Nephrol. 2016;48(4):585-91.

20. Berger TG, Steinhoff M. Pruritus and renal failure. Semin Cut Med Surg 2011;30(2):99100.

21. Chen HY, Chiu YL, Hsu SP,Pai MF, Lai CF, Yang JY. Elevated C-reactive protein level in hemodialysis patients with moderate/severe uremic pruritus: a potential mediator of high overall mortality. QJM 2010;103:837-846.

22. Fallahzadeh MK, Roozbeh J, Geramizadeh B, Namazi MR. Interleukin-2 serum levels are elevated in patients with uremic pruritus: a novel finding with practical implications. Nephrol Dial Transplant 2011;26:3338-334.

23. Takahashi T, Kubota M, Nakamura T, Ebihara I, Koide H. Interleukin-6 gene expression in peripheral blood mononuclear cells from patients undergoing hemodialysis or continuous ambulatory peritoneal dialysis. Ren Fail 2000;22:345-354.

24. Subach RA, Marx MA.Evaluation of uremic pruritus at an outpatient hemodialysis unit. Ren Fail. 2002;24(5):609-14.

25. Szepietowski JC, Balaskas E, Taube KM,Taberly A, Dupuy P. Quality of life in patients with uremic xerosis and pruritus. Acta Derm Venerology 2011;91(3):313-317. 